\title{
Chaotic behavior of seismic mechanisms: experiment and observation
}

\author{
Bento Caldeira*, Hugo G. Silva, José F. Borges, Mouhaydine Tlemçani, Mourad Bezzeghoud
}

Universidade de Évora, Escola de Ciências e Tecnologia, Centro de Geofisica de Évora e Departamento de Física, Évora, Portugal

\author{
Article history \\ Received August 9, 2011; accepted February 28, 2012. \\ Subject classification: \\ Earthquake faults: properties and evolution, Earthquake source and dynamics, Seismic risk, Earthquake modeling, Chaos theory.
}

\begin{abstract}
To simulate the dynamics of earthquakes, a mechanical prototype was constructed that was inspired by the Burridge-Knopoff model and equipped with accurate instrumental devices. The data obtained by the prototype appeared to be consistent with seismic data from the San Andreas Fault, California, USA, which were analyzed using two different methodologies: seismology and modern developments of chaos theory. Perspectives for future work are also presented.
\end{abstract}

\section{Introduction}

Modeling the dynamics of failure would uncover important aspects of seismology and earthquake prediction if it can account of all of the variables. However, given the complexity of the systems involved, earthquake prediction is difficult, even when performed in well-monitored areas such as the San Andreas Fault (SAF; California, USA). Nevertheless, this subject should not be ignored, and new perspectives should be tested. Although simplified, the predicted data might be analogous to those of the actual faults. In this context, simple models with dynamics that are qualitatively similar to those of active faults need to be developed. Such models can allow for the identification of specific signatures that are observed prior to major earthquakes, and if these can be cautiously applied, this might limit the human loss.

In the 1960s, Burridge and Knopoff (BK) introduced the mechanical block-spring model [Burridge and Knopoff 1967] to simulate interactions between two surfaces of a fault. This was the first step in earthquake modeling. Their model was based on the principle of 'elastic rebound', which considers a differential shift between the two sides of an active fault in an expanded time scale, elastic deformation in geological materials, and an increase in stress. When the accumulated stress exceeds the limit of the forces that oppose failure (mainly friction), the links between both sides break, which triggers a rapid release of the stress. This then reshapes the deformed material, and causes an earthquake. From the viewpoint of classical physics, seismic phenomena appear as a discontinuity in the junction of two elastic half-spaces that are subjected to constant deformation. This model, which is supported by the electrodynamic theory proposed by Volterra [1907] and modified by Love [1944], is the basis of seismic source theory, which accurately describes the surface displacement field that is associated with an earthquake, although it fails when applied to the prediction of seismic cycles.

The BK model is illustrated in Figure 1, and it consists of a set of blocks that are linked by springs and placed onto an unpolished surface. Each block of the 'train' is connected through another spring to a sliding surface that moves slowly at a constant speed. When the forces on the blocks are less than the maximum force of static friction, the blocks remain at rest. However, the springs that attach the blocks to the sliding surface accumulate stress, which increases the amount of force applied to each block. Once the block overcomes the solid friction force, it slips, which unburdens the spring that connects the block to the sliding surface and loads the springs that couple the block to contiguous blocks, triggering their movement. Each rebound is equivalent to an earthquake. A small-amplitude rebound that occurs with only one of the blocks is correlated to a minor earthquake. However, a rebound that drags the other blocks and causes an avalanche of rebounds is considered to be a major earthquake. The system that is used to model seismic faults can be represented by the superposition of two processes that run on different time scales. Specifically, the accumulation of stresses is a slow and continuous process, whereas the release of the stress is a discontinuous and fast process. These processes sum up earthquake dynamics.

Indeed, since the introduction of the BK model, earthquakes have been primarily studied using numerical calculations. Such simulations can successfully describe many properties of earthquakes accurately. For a review of this topic, the reader is referred to Carlson et al. [1994]. Nevertheless, only a handful of experimental approaches have been performed [e.g., Feder and Feder 1991], and two different types of solutions have been found. When the Coulomb friction law is applied and the system is assumed to be symmetric, periodic behavior is obtained. In contrast, when 


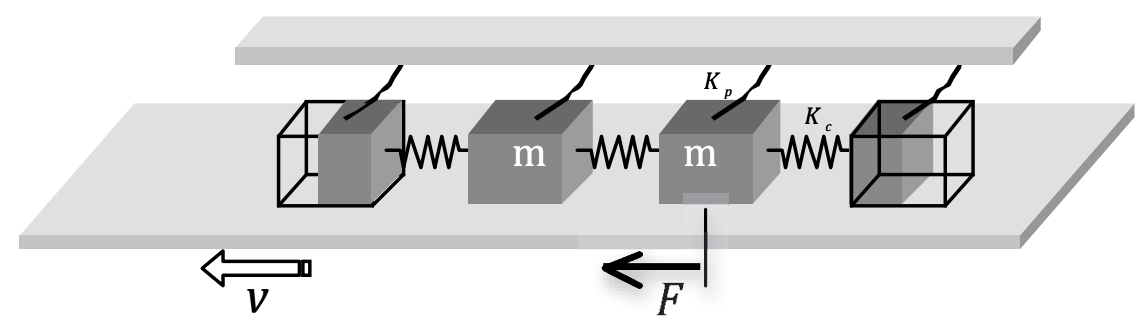

Figure 1. Diagram of the Burridge-Knopoff mechanical model. The system is composed of equal masses $(m)$ that are connected to the system by horizontal springs of strength $k_{c}$ and vertical springs of strength $k_{p}$. The mass is subjected to the force of friction $F(x)$, which only depends on the velocity of the block $(V)$.

laws other than that of Coulomb are applied or symmetry is not considered in the model, the solutions are in agreement with the existence of a rebound distribution, and they obey a power law. This power law is such as that observed in the distribution of earthquake magnitudes, which corresponds to the Gutenberg-Richter law [Gutenberg and Richter 1956].

Recent developments in 'chaos theory' have been observed in the BK model. For instance, Hahner et al. [1998], Vieira [1999], Rubeis et al. [1996], Hallgass et al. [1997], Cartwrifht et al. [1997], and Field et al. [1995] obtained results that are consistent with observations such as Omori's law [Shcherbakov et. al. 2004]. Among these approaches, the concept of 'self-organized criticality' [Bak et al. 1987], which is derived from statistical physics, has contributed significantly. These models attempt to describe the behavior of thermodynamic systems that evolve spontaneously into critical states of equilibrium that are subjected to fluctuations that are characterized by statistics that follow fractal relations, which is also known as self-organized criticality behavior. Accordingly, seismic phenomena are the result of relaxation processes induced by small perturbations in the Earth crust that have obtained a 'metastable' equilibrium. The recognition of self-organized criticality behavior in seismic models has been tested using numerical simulations [Vilotte et al. 1994, Hahner and Drossinos 1998, Vieira 1999, Rubeis et al. 1996, Hallgass et al. 1997] and in electronic circuits called 'cellular automata' [Cartwrifht et al. 1997, Field et al. 1995]. In all cases, the modulations originated in the equations of motion governing mechanical systems that consist of chains of blocks and springs, all of which are inspired by the BK model. Evidence of chaotic behavior has been detected in seismic models; thus, based on these observations, it can be concluded that actual 'seismic faults' show the same type of behavior.

In the present study, we obtained a novel view of seismic phenomena by assessing the subject in two different ways. As described in Section 2, a homemade 'earthquake machine' (EQM) was developed and evaluated, and the seismicity of the SAF was analyzed, as presented in Section 3. The results of both approaches are discussed in Section 4, and some final remarks are provided in Section 5.

\section{The earthquake machine}

For the experimental portion of the present study, the EQM shown in Figure 2 was designed. The mechanical prototype consisted of a rubber conveyor carpet that was actuated by a stepping motor with an electronic speed controller, on which several blocks connected by springs were placed. However, in this case, only one block was used. When the machine was operated for a period of time, cycles of stress accumulation and release were accompanied by slow movement of the block in relation to the carpet. We equipped the springs of the EQM with a set of force sensors that were connected to a data-acquisition system, which was linked to a computer. The data recorded by the prototype consisted of temporal changes in the electrical voltage, which were converted to mechanical stress in the springs (although the knowledge of the 'true' mechanical stress is totally irrelevant for this analysis), which was measured by sensors that corresponded to the sequence of stress accumulation and drops in the springs. Figure 3a shows an extract from the temporal series that was used in the present study.

Data processing was performed according to two distinct methodologies. The first analysis treated the data according to the techniques used in seismology with field measurements (real data). To this end, an event was considered to occur when a drop in stress was observed. The magnitude of the event was calculated from the value of the stress drop. Following this approach, a given stress drop was related to a respective magnitude. In the second methodology, we used the series recorded in the context of chaos theory to investigate the behavior of the prototype.

The first analysis of the data produced by the machine was designed according to the methodology used in seismology. Stress drops were identified in the series, and their values were calculated. Considering that $\sigma_{i}$ is the measured stress at a given instant $\left(t_{i}\right)$, stress drops were calculated as: $\Delta \sigma_{i}=\sigma_{i}-\sigma_{i+1}$. The values of the stress drops were positive, and the relationship between the stress drop and the magnitude was obtained according to the method of Kanamori and Anderson [1975], who applied a rectangular model of faulting: 

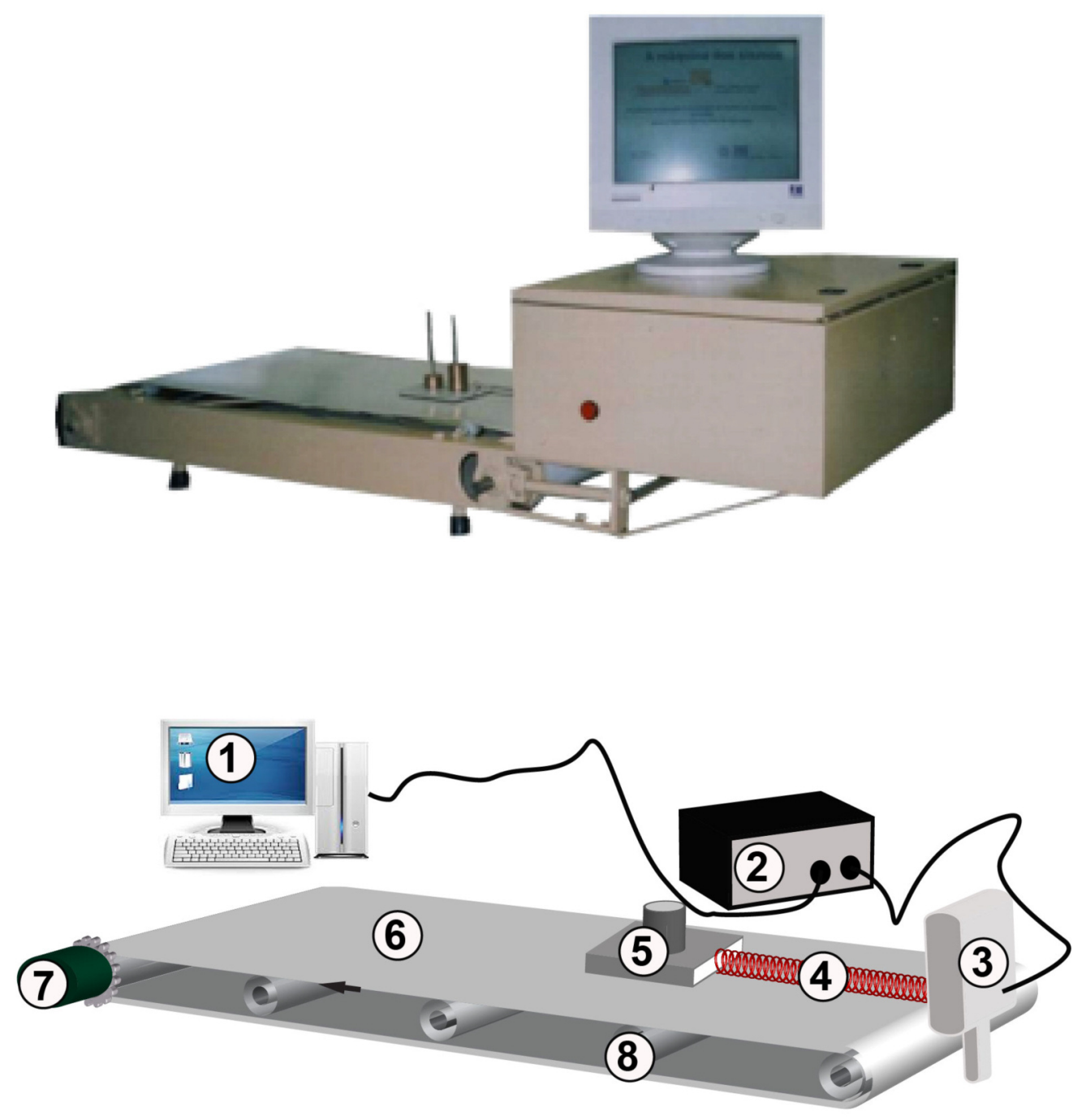

Figure 2. Top: Photograph of the earthquake machine prototype. The left part contains the mechanical components, including the carpet and the block that is connected to the spring. The right side of the apparatus contains the control box, which consists of a computer acquisition system, a force sensor, and a motor control. Bottom: Schematic representation of the earthquake machine prototype: (1) personal computer; (2) electronic interface; (3) forcesensing; (4) spring; (5) block; (6) rotating carpet; (7) motor; (8) rotating drum.

$$
M=C+\frac{2}{3} \log \Delta \sigma
$$

where $C$ is an empirical constant, the value of which was selected to normalize the magnitude of the EQM events to those of real earthquakes. In this sense, to determine $C$, we considered that the average stress drop $\langle\Delta \sigma\rangle$ should correspond to an earthquake with a magnitude of 3.7. As a result, $C=3.7-2 / 3 \log \langle\Delta \sigma\rangle$. According to this procedure, magnitudes that corresponded to stress drops greater than the numerical noise level that was introduced by the acquisition system were calculated.

Figure 4a represents the distribution of event numbers (arranged in five magnitude classes). As shown in Figure 4a, the Gutenberg-Richter law $(\log (N)=a+b M$, where $N$ is the number of seismic events with magnitudes in the range of $M \pm \Delta M / 2)$, fits the experimental data well. The values of $a$ and $b$ are presented in the plot and are compared to the actual seismicity (Figure 4b) in Section 5.
In addition, the observations provided several clues regarding the chaotic behavior of the system. This perspective was used in the second EQM data analysis. Chaotic character in a dynamic system is usually identified by representing the evolution of the system in the phase space, in which each point represents a state of the system. If the path has fractal geometry, it is called a 'strange attractor', and the system shows chaotic dynamics. However, these representations are often impossible to produce when the number of coordinates that are required to represent a state of the system is greater than three. Therefore, instead of representing the trajectories in the phase space, we can calculate some of its properties, such as the fractal dimension (D), which can be determined for any number of degrees of freedom. In addition, the system dynamics must be rebuilt in the phase space, and the scalar series of measurements must come from only one quantity. In such cases, reconstruction of the phase space [Goltz 1998] can be 

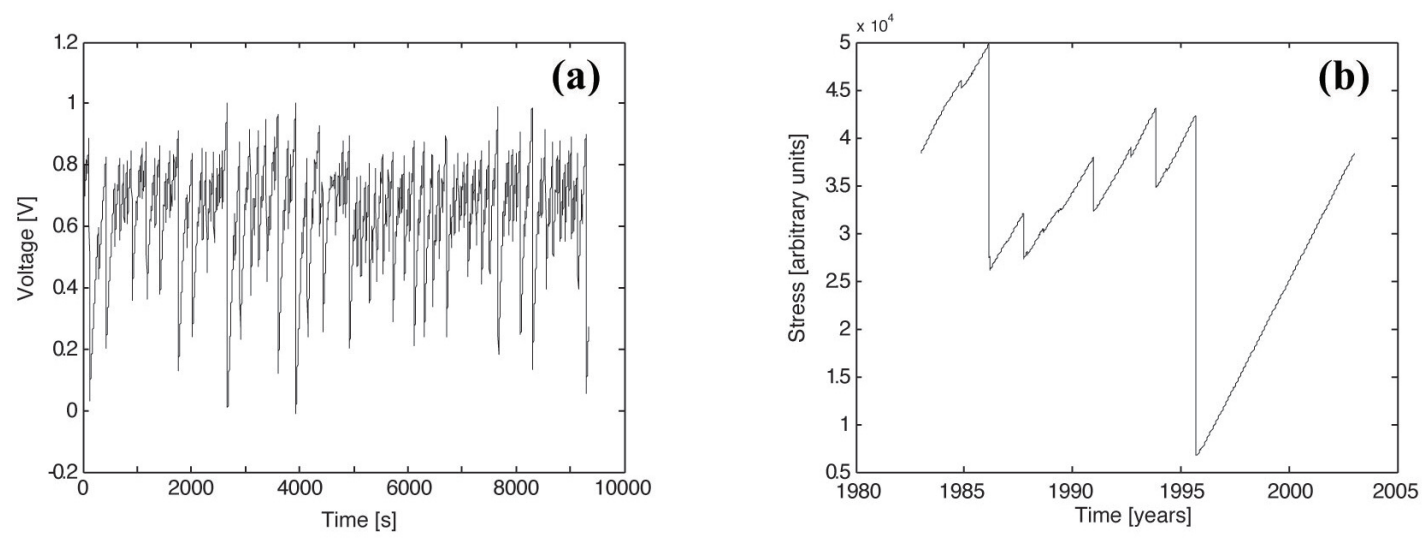

Figure 3. (a) Extracts of the time series obtained by monitoring the earthquake machine at a rate of 10 samples per second. (b) Time series reconstructed from 20 years (1980-2000) of SAF seismicity.
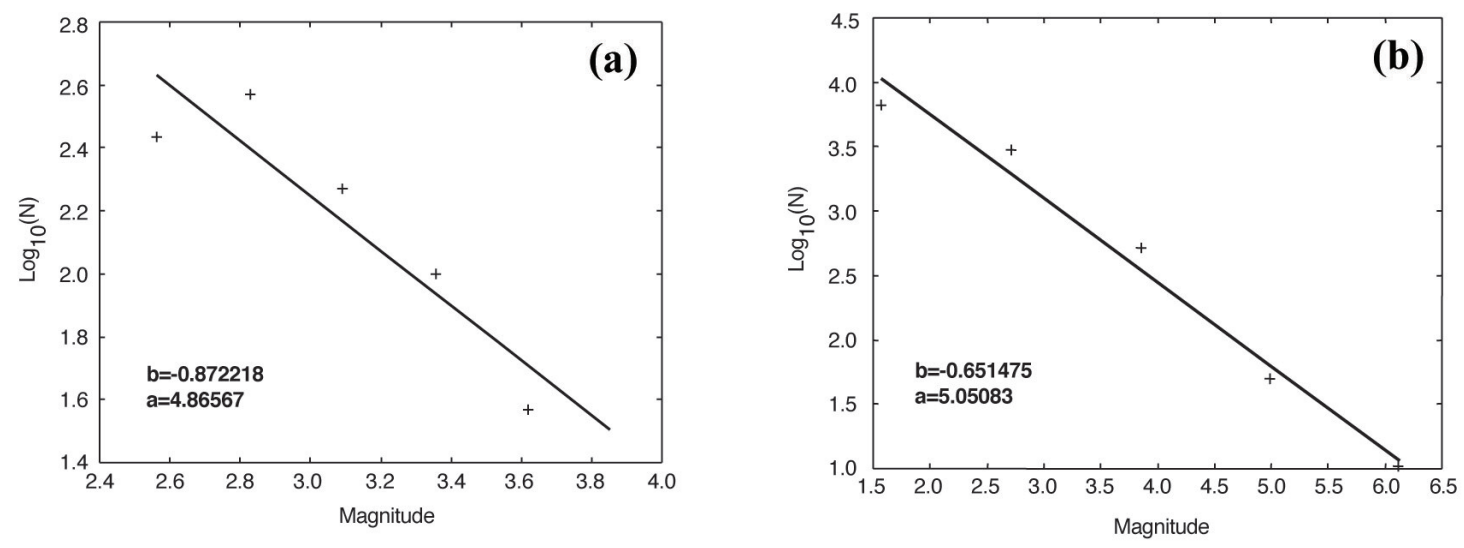

Figure 4. (a) Gutenberg-Richter distribution of events recorded with the prototype during a series of 736 events. (b) Gutenberg-Richter distribution of earthquakes recorded in California between 1980 and 2000 (10,256 events).

achieved through a technique called 'suspension', which involves the introduction of a parameter that designates the 'suspension order', which is also known as the 'embedding dimension' (d). With this dimension, a time-delay reconstruction of the system phase space provides the necessary number of coordinates to unfold the dynamics from overlaps upon itself that are caused by projection. Usually, to determine the fractal dimension $(D)$ of the attractor, the embedding dimension is required to reconstruct a space that is equivalent to the phase space of the system.

In practice, obtaining the fractal dimension of the attractor made from the scalar time series is a process that involves the successive construction of growing phase spaces (reconstitution dimension), and the calculation of fractal dimensions that are associated with each of the phase spaces. The time series is derived from a chaotic dynamic system; thus, if the fractal dimension does not increase after a certain order of reconstitution, i.e., if the function $D(d)$ becomes saturated, the fractal dimension is the embedding dimension. The calculation of the fractal dimension that is associated with each reconstituted space requires the use of appropriate software. In this case, we used the FD3 program [Serraille 1992], which was written according to the algorithm known as 'box counting'.

The number of parameters required to model the dynamic system under study can be estimated using the fractal embedding dimension [De Santis 1997, Kovalenko et al. 2008]. In general, the value of the reconstitution dimension for which $D$ is saturated gives an estimate of the minimum number of variables that are required to model the system, and $2 D+1$ provides an estimate of the maximum value.

By combining well-known relations of seismology with an equation that quantitatively defines a fractal set [Turcotte 1997], we obtain the following theoretical relationship:

$$
D=2 b
$$

which relates the fractal dimension $(D)$ to the $b$-value of the Gutenberg-Richter law. Thus, from the Gutenberg-Richter analysis of the data, we found that $D \approx 1.74$.

Interestingly, the reconstruction of phase space dimensions using reconstitution dimensions $(d=1,2$ and 3$)$ enables us to obtain $D=1.73$ for $d=3$, which is in close agreement with the value that was obtained from the above relationship. 


\section{Seismicity of the San Andreas Fault}

In parallel to the previous data analysis, to compare the time series obtained by the EQM to those observed in an actual seismic region, we analyzed the instrumental seismicity in a region near the SAF. We considered 10,256 events in the area over a period of 20 years (1980-2000). The reconstruction of the series was based on two assumptions. The first assumption is that the stress balance is equal to zero throughout the 20 years of monitoring; i.e., in the study region, the sum of stress released is equal to the sum of the accumulated stress. The second assumption is that the accumulation of stress occurs at a constant rate. Based on these two assumptions, using the times and magnitudes of the events, the magnitudes were transformed into stress drops, enabling calculation of the temporal coefficient of stress accumulation, which was achieved by summing the stress drops. In combination with the timing of various events and corresponding stress drops, this coefficient allowed us to reconstruct the time series shown in Figure $3 \mathrm{~b}$. As can be seen from the presented series, the corresponding chart is slightly different from the series that was obtained by the EQM. In particular, the data derived from the EQM were represented on a scale that covers the entire dataset, and only the five largest stress drops from all of the 10,256 events could be distinguished in the SAF data. The rest of the events can only be clearly observed on a smaller scale. Moreover, magnification of small segments of the graph were indistinct compared to the global representation; thus, due to the fractal nature of the seismic activity in the study region, the pattern of the series is preserved when viewed on a scale that was compatible with the five orders of magnitude upon which the data were distributed. This finding was also observed in the data that were obtained from the EQM; however, the effect is attenuated by the reduced discrimination of 'seismic events' allowed by the instrumentation. Indeed, an inspection of the fractal dimension of the SAF seismicity revealed its complex nature, which indicated that the multi-fractal behavior of the SAF data was relatively complex compared to the results of the $\mathrm{EQM}$, as discussed in the next section.

\section{Discussion}

Interesting similarities were observed in the analysis of the EQM and SAF seismicity. The value of $b$ was 0.87 for the EQM and 0.65 for the SAF, which are comparable values. Moreover, both systems show fractal behavior. The EQM had a $D$ factor of 1.73 , whereas the seismicity of the SAF tended to show more complex behavior due to the occurrence of multi-fractals, which will require further study in the future.

Although the characteristic spatial (centimeters for EQM, and kilometers for the SAF) and temporal (seconds for EQM, and decades for the SAF) units of the EQM and the
SAF data are different, the results show that the dynamics of both systems are similar. Although the EQM is simple, it successfully captures earthquake dynamics and might provide evidence to inform future studies.

\section{Final remarks}

The present study, which is the first step towards a deeper analysis of seismic mechanisms in the context of chaos theory, reveals several interesting results. As discussed in Section 4, although simple in nature, the present prototype provided trends that were similar to those of the actual data and it might uncover important aspects of actual seismicity. Various improvements, such as the consideration of two blocks and different carpets, will be applied in future studies, to determine key seismic signatures that occur before major earthquakes. The proposed prototype could improve our understanding of different seismic precursors, such as seismo-electromagnetic phenomena [Silva et al. 2011].

Acknowledgements. This study was developed with the support of the Agencia Nacional Ciência Viva of the Ministério para a Ciência, Tecnologia e do Ensino Superior (MCTES) through the project EXPER POCTI/DIV/2005/00047. HGS acknowledges support from the Science and Technology Foundation for project no. SISMOD/LISMOTPTDC/CTE-GIN/82704/2006 and grant no. SFRH/BPD/63880/2009, and thanks the Calouste Gulbenkian Foundation for the Estímulo à Criatividade e à Qualidade na Actividade de Investigação Award of 2010. The authors are thankful to Bruno Romeira and José Figueiredo for their important discussions on chaos theory. The support of Sara Fernandes is also gratefully acknowledged.

\section{References}

Bak, P., C. Tang and K. Wiesenfeld (1987). Self-organised criticality: An explanation of the 1 / f noise, Phys. Rev. Lett., 59, 381-384.

Burridge, R., and L. Knopoff (1967). Model and theoretical seismicity, B. Seismol. Soc. Am., 57 (3), 341-371.

Carlson, J.M., J.S. Langer and B.E. Shaw (1994). Dynamics of earthquake faults, Rev. Mod. Phys., 66, 657-670.

Cartwrifht, J., E. Garcia and O. Piro (1997). Burridge-Knopoff models as elastic excitable media, Phys. Rev. Lett., 79, 527530.

De Santis, A. (1997). A direct divider method for fractal selfaffine profiles and surfaces, Geophys. Res. Lett., 24, 20992102.

Feder, H.J.S., and J. Feder (1991). Self-organized criticality in a stick-slip process, Phys. Rev. Lett. 66, 2669-2672.

Field, S., N. Venturi and F. Nori (1995). Marginal stability and chaos in stick slip electronic circuit modelling coupled faults, Phys. Rev. Lett., 74, 74-77.

Goltz, C. (1998). Fractal and Chaotic Properties of Earthquakes, Springer, $178 \mathrm{pp}$.

Gutemberg, B., and C.F. Richter (1956). Magnitude and energy of earthquakes, Annali di Geofisica, 9 (1), 1-15; republished in Annals of Geophysics, 53 (1), 2010, 7-12, doi:10.4401/ag-4588. 
Hahner, P., and Y. Drossinos (1998). Nonlinear dynamics of a continuous spring-block model of earthquake faults, J. Phys. A-Math. Gen., 31, L185.

Hallgass, R., V. Loreto, O. Mazzellla, G. Paladin and L. Pietronero (1997). Earthquake statistics and fractal faults, Phys. Rev. E., 56, 1346-1356.

Kanamori, H., and D. Anderson (1975). Theoretical basis of some empirical relations in seismology, B. Seismol. Soc. Am., 65, 1073-1095.

Kovalenko, V.V., E.V. Gaidukova and A.B.G. Kuassi (2008). Fractal river-flow diagnostics for stable description of long-term variations of hydrologic characteristics, Russ. Meteorol. Hydrol., 33, 247-252.

Love, A.E. (1944). Treatise on the Mathematical Theory of Elasticity, 4th ed., New York, 643 pp.

Rubeis, V., R. Hallgass and V. Loreto (1996). Self-affine asperity model for earthquakes, Phys. Rev. Lett, 76, 25992602.

Serraille, J. (1992). Developing Algorithms For Measuring Fractal Dimension, public ftp-server: csustan.csustan.edu. Shcherbakov, R., D.L. Turcotte and J.B. Rundle (2004). A generalized Omori's law for earthquake aftershock decay, Geophys. Res. Lett., 31, L11613.

Silva, H.G., M. Bezzeghoud, J.P. Rocha, P.F. Biagi, M. Tlemçani, R.N. Rosa, M.A. Salgueiro da Silva, J.F. Borges, B. Caldeira, A.H. Reis and M. Manso (2011). Seismo-electromagnetic phenomena in the western part of the Eurasia-Nubia plate boundary, Nat. Hazards Earth Syst. Sci., 11, 241-248.

Turcotte, D.L. (1997). Fractals and Chaos in Geology and Geophysics, Cambridge University Press, 2nd. ed., 416 pp.

Vieira, M. de Sousa (1999). Chaos and synchronised chaos in an earthquake model, Phys. Rev. Lett., 82, 201-204.

Vilotte J.-P., J. Schmittbuhl and S. Roux (1994). Some physical properties of the Burridge-Knopoff model. Lecture Notes in Physics, In: K.K. Bardhan, B.K. Chakrabarti and A. Hansen (eds.), Non-Linearity and Breakdown in Soft Condensed Matter (proceedings of a workshop held at Calcutta, India, 1-9 December 1993), 437, 54-77.

Volterra, V. (1907). Sur L'equibre Des Corps Elastiques Multiplement Connexes, Ann. Sci. École Normal Superieur de Paris, 24, 401-517.

\footnotetext{
${ }^{\star}$ Corresponding author: Bento Caldeira,

Universidade de Évora, Escola de Ciências e Tecnologia,

Centro de Geofisica de Évora e Departamento de Física, Évora, Portugal; email: bafcc@uevora.pt.

(C) 2012 by the Istituto Nazionale di Geofisica e Vulcanologia. All rights reserved.
} 